\title{
A Fault Feeder Selection Method Based On Transient Amplitude Fault Measure
}

\author{
Guangtai YAN, Jiawen LIANG, Xintao WANG, Anhua LIU, Yang BAI \\ Liaocheng Power Supply Company, Shandong Liaocheng 252000, China
}

\begin{abstract}
In order to accurately detect single-phase resonant network grounding fault, puts forward a fault line selection method based on fault transient amplitude measurement. Through the analysis of fault feeder modulus transform, after occurrence of a fault and normal feeder, using wavelet modulus maxima characteristics of current zero mode, obtained initial current wavelet modulus maxima of law. Combining the definition of the law and the general fault measure, defined the transient amplitude fault measurement. The amplitude of the transient fault feeder fault measure maximum amplitude, transient fault measure normal feeder is greater than 0 and close to 0 . According to detect the fault feeder. Theoretical analysis and ATP simulation results show that, this method can effectively and accurately detect single-phase resonant network grounding fault, not influenced by arc suppression coil, it proves the correctness of this method.
\end{abstract}

KEYWORD: resonant grounding system; single-phase ground fault; zero mode initial current; transient amplitude fault measure; ATP simulation

\section{INTRODUCTION}

Small current grounding mode is widely used in power distribution network in china. Weak singlephase grounding fault current is superimposed onto a larger load current, it is difficult to distinguish the resonant power system fault location feeder[1-3]. Therefore, research on fault line selection technique is sensitive and reliable imminent. In recent years, fault line selection method based on measurement can determine the fault feeder, but it doesn't matter in-depth excavation of the transient component, the sensitivity is not high [4,5].

Firstly, using wavelet modulus maxima description of the current key defined transient amplitude fault measure, using this measure to distinguish normal fault feeder and feeder. Puts forward the line selection method. ATP simulation and verification: this fault line selection method is correct.

\section{THE DEFINITION OF FAULT TRANSIENT AMPLITUDE MEASUREMENT}

Fault measure (Fault Measures) refers to a characterization of each feeder failure possibility of positive real number in a criterion reference. At the same voltage feeder level network, if the fault measurement of a feeder maximum, can show that the feeder for fault feeder possibility is biggest.

Put forward the concept of fault measures that result is output symbol for improving results of numerical output type. In the fault feeder uncertain circumstances, the same voltage grade all feeder are doubtful. May be influenced by the interference of feeder fault of small current grounding fault line selection criterion of judgment, and may not ensure a line must be fault feeder. The concept of fault measures into the line selection method of small current grounding fault, to optimize the fuzzy judgment results, in order to improve the reliability of the results of line selection.

In this paper, by the modulus maxima of wavelet transform zero mode fault current characteristics highlighted, tectonic fault transient amplitude measurement.

Assuming a neutral non effective grounding grid consists of feeder, make the said article feeder for amplitude measurement, the zero mode initial current traveling wave characteristics, small current grounding fault of wavelet transform theory and concept of fault measures good combination, structure of travelling wave amplitude measurement.

By the concept of fault measurement requirements knowable, travelling wave amplitude measurement indicates the possibility of fault occurred in the feeder. Therefore, the scope of 
travelling wave amplitude measure defined in.

Travelling wave amplitude measure Ruo Di feeder, then the fault occurred in the feeder is a possibility, that is to say the sound feeder feeder.

Travelling wave amplitude measurement of Ruo Di feeder, then the fault occurred in the feeder of the possibility, that is to say the feeder fault feeder.

Travelling wave amplitude measure the Ruo Di feeder and very close, then the fault occurred in the feeder of the possibility of very large, that is to say the feeder is a great possibility of fault feeder.

Travelling wave amplitude measure the Ruo Di feeder and very close, then the fault occurred in the feeder of the possibility is very small, that is to say the feeder is a great possibility of sound feeder.

With the first a wavelet transform modulus expressed the line after failure of initial current traveling wave zero modulus maxima. Due to numerical wavelet transform modulus maxima is divided into positive and negative, but not for the modulus maximum value, and thus. After failure, the line of zero mode amplitude of initial current traveling wave first modulus maximum value of wavelet transform used to said, i.e..

Occurred in the first line hypothesis of singlephase ground fault, zero mode initial current traveling wave wavelet modulus maxima of the feeder amplitude with said. According to the second chapter of the theoretical analysis and wavelet transform theory, and the zero mode initial current traveling wave wavelet modulus maxima compared to other feeder amplitude, maximum, and satisfy the relation:

$$
\begin{aligned}
& M(k)=(N-1) M(j) \\
& \text { Sum }=\sum_{j=1}^{N} M(j) \quad j \in[1, N \\
& \text { Sum }=2 M(k) \\
& C_{1}(j)=\frac{M(j)}{S u m} \\
& C_{2}(j)=\frac{1}{4}\left\{1+\frac{1}{N-1} \sum_{l=1, l \neq j}^{N} \operatorname{sgn}[M(j)-M(l)]\right\} \\
& \operatorname{sgn}(x)=\left\{\begin{array}{cc}
1 & x>0 \\
0 & x=0 \\
-1 & x<0
\end{array}\right. \\
& C(j)=C_{1}(j)+C_{2}(j)
\end{aligned}
$$

\section{SIMULATION OF FAULT LINE SELECTION METHOD}

Simulation of feeder 2 , and in the single-phase grounding transient resistance, that is, the initial phase angle for, and. Due to limited space, only the given waveform as shown in figure 3. Amplitude measurement as shown in table 1 .

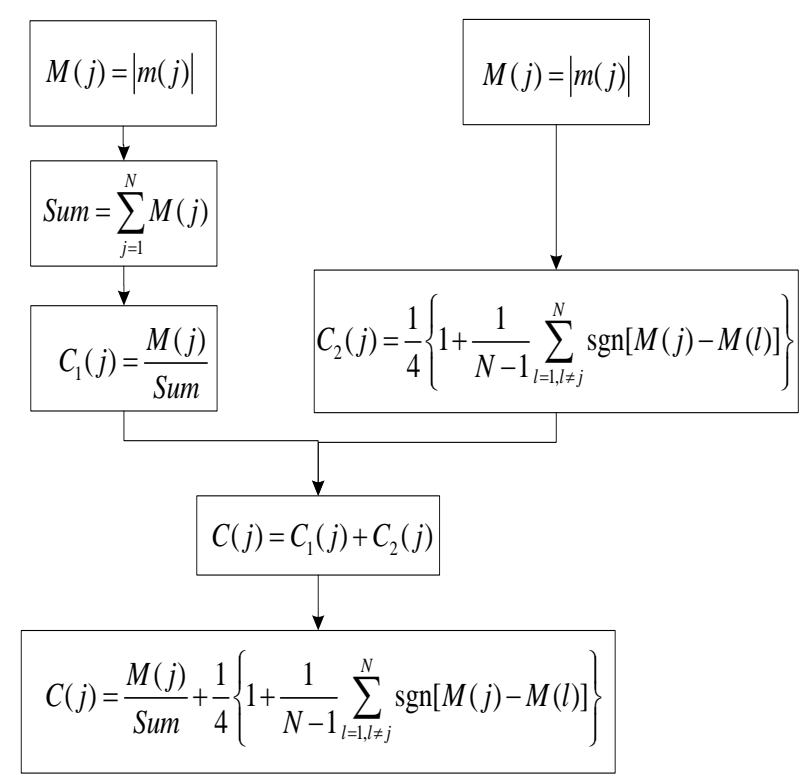

Fig. 1 Schematic structural amplitude measurement

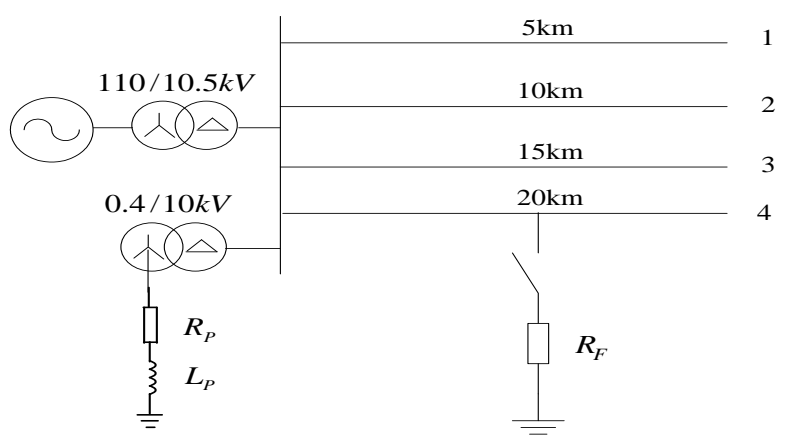

Fig. 2 ATP simulation of resonant grounding system


Fig.3 4 zero modulus current wavelet coefficients of $30^{\circ}$ initial phase 


\section{SUMMARY}

It has important meaning to study the techniques of line selection of single phase to ground fault in distribution network. This article from the concept of fault measure, define the amplitude range of fault transient fault measure, measure is [ $\left[\begin{array}{ll}0 & 1\end{array}\right]$. Measure the nearest 1 feeder for fault feeder. The method is simple, and is suitable for the resonant earthed system is worth popularizing.

Table1 line transient amplitudes of fault feeder 2 faulting

\begin{tabular}{|c|c|c|c|c|}
\hline measure & Line 1 & Line 2 & Line 3 & Line 4 \\
\hline $30^{\circ}$ & 0.1998 & 0.9998 & 0.5002 & 0.3501 \\
\hline $60^{\circ}$ & 0.3501 & 0.9971 & 0.3501 & 0.3501 \\
\hline $90^{\circ}$ & 0.3000 & 1.0002 & 0.3000 & 0.3000 \\
\hline
\end{tabular}

\section{REFERENCES}

[1] ZHANG Pujun, ZHOU Yiquan. Discussion of high impedance fault. Fujian Power System and Electric Engineering, 2002, 22(12): 17-18.
[2] SHI Shenxing, DONG Xinzhou, ZHOU Shuangxi. Analysis of single phase to ground fault generated traveling waves. Automation of Electric Power Systems, 2005, 29(23): 2932.

[3] MAO Peng, YANG Li, DU Xiaogong. Study of the distance relay based on zero sequence components. Automation of Electric Power Systems, 2003, 27(10): 6062.

[4] CHEN Xiangxun. Some basic concepts of wavelet. Automation of Electric Power Systems, 2004, 28(1): 125.

[5] SU Pengsheng, WANG Huan. Discussion of the short2window Morlet component wavelet algorithm on the power system signal process. Automation of Electric Power Systems, 2004, 28(9): 36-42.

\section{ABOUT THE AUTHOR}

YAN Guangtai (1989-), male, master of engineering, assistant engineer, mainly engaged in power system operation and control and relay protection E-mail: $312844901 @$ qq.com 